\title{
Utilización de indicadores como respuesta a la introducción de la sostenibilidad en las ciudades mexicanas en el s.XXI.
}

\author{
Alfred Esteller Agustí1, Isamar Anicia Herrera Piñuelas², \\ Adolfo Vigil de Insausti ${ }^{3}$ \\ ${ }^{12}$ Universidad del Valle de Atemajac (UNIVA), Jalisco, México. \\ ${ }^{3}$ Departamento de Urbanismo, Universidad Politécnica de Valencia, Valencia, España \\ E-mail: ${ }^{1}$ alfred.esteller@univa.mx, ${ }^{2}$ isamar.herrera@univa.mx, ${ }^{3}$ advide@urb.upv.es
}

\begin{abstract}
Resumen. Debido al mayor acceso a la información gracias al incremento de acceso a las nuevas tecnologías de información y comunicación (nTIC) la sociedad mexicana se encuentra con un acceso a la información nunca visto hasta el siglo XXI. En este contexto, la sociedad se encuentra con a través de las nTIC con multitud de opiniones y datos que pueden ser poco contrastados, propiciando una menor profundización en los saberes, reduciendo la posibilidad de argumentar un criterio propio y un menor control sobre los conocimientos técnicos específicos, en contraste con los saberes ancestrales y transmitidos de generación en generación, entre los que se puede incluir la forma de hacer ciudad así como la arquitectura vernácula. En la actualidad, conceptos como calentamiento global, ecología y sustentabilidad son tema de discusión en cualquier ámbito, mientras que, a distancia, se denota carencia de conocimiento del mismo. En la búsqueda de un respaldo cientifico, se concluye que el mejor camino para introducir el tema de sostenibilidad al proceso de diseño, construcción y ciudad es mediante Indicadores de sostenibilidad, presentándose como la herramienta fundamental, medible y comprobable para la creación de parámetros que fundamenten los pilares de la sostenibilidad, una arquitectura económicamente viable, socialmente responsable y ambientalmente afable.
\end{abstract}

Palabras clave: sostenibilidad, edificación, indicadores, ciudad.

\section{Introducción}

El comportamiento humano del último siglo, no es el que históricamente ha venido desarrollando el mayor cambio en el daño ocasionado al entorno natural, aunque sí se encargó de recoger un proceso paulatino que comenzó en el siglo XVIII con todas las evoluciones que trajo consigo la Revolución Industrial, y en la difusión de éstas a lo largo y ancho de todo el planeta, con diferentes repercusiones en cada lugar, y no siempre beneficiosas en lo que a México y el resto de América latina corresponde, debido a su condición de países en vías de desarrollo. Pero analizando las actividades humanas nos encontramos en la situación de que indudablemente el siglo XXI arrastra ya dos siglos con programas, materiales y sistemas que vienen deteriorando la salud ambiental y por consiguiente el bienestar humano, siendo uno de los campos más influyentes el de la construcción y desarrollo de ciudades, un entendimiento de las implicaciones en las etapas, puede venir a incidir en un entendimiento científico de las consideraciones en el proceso de edificación.

\section{Contexto histórico}

La arquitectura vernácula como explica R. M. Sánchez; “...Surge como sintoma de la realidad de un pueblo bien definido, representa su devenir histórico, sus circunstancias culturales y la sintesis de sus origenes e influencias..." (Sánchez Lara. 1980:11), legados de sostenibilidad de las distintas civilizaciones, 
Fig. 1. Diagrama ámbitos de la sostenibilidad. Representación evolucionada con los tres pilares de la sostenibilidad, ambiental, social y económico, más la incorporación de una base cultura, vinculación política $\mathbf{y}$ apoyo técnico. Fuente. Elaboración propia

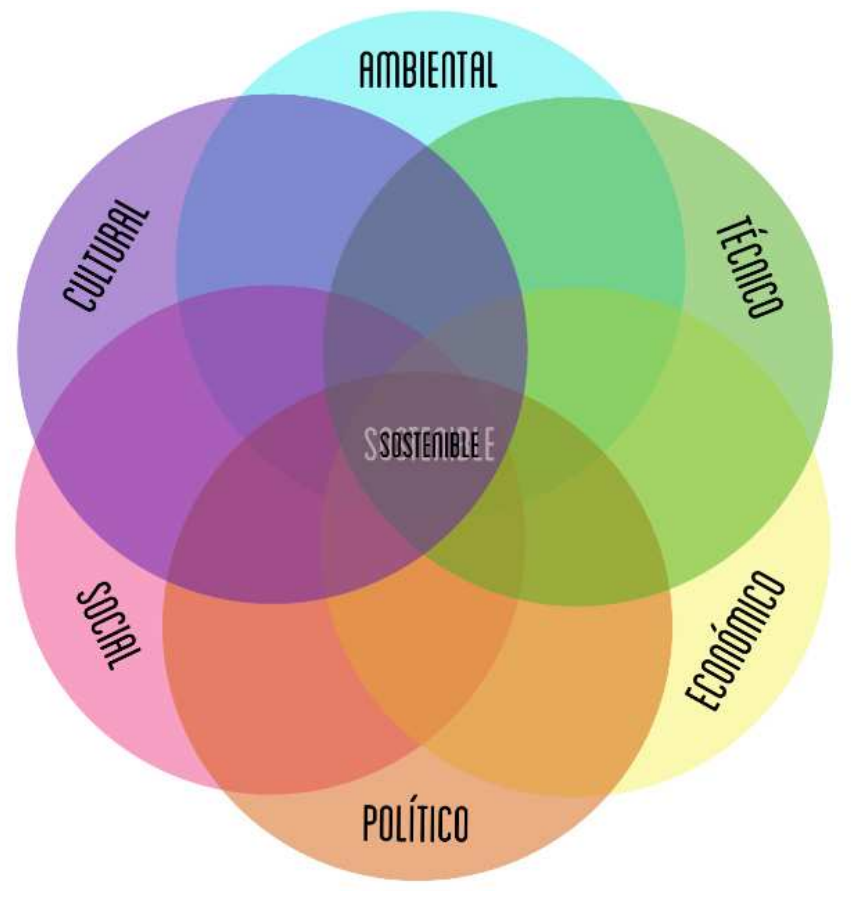

sociedades y pueblos, como recoge J. Taylor "En la mayoría de las viviendas primitivas se detecta una profunda consideración de las condiciones locales; sus constructores, por necesidad, aprovechan al máximo accidentes naturales para aumentar la comodidad $y$ protección" (Taylor. 1984:9).

Citando de nuevo a R. M. Sánchez; "En cuanto a la fisonomía de los pueblos vernáculos, mantienen cierta unidad y gran congruencia con el entorno (...) ya que los materiales usados en la construcción como el adobe, tablas, tejas, hojas de palma, cactus, palapa, etc., son productos que en su base ofrecen el medio fisico y cuya manufactura es posible a través de modos preindustriales y repetitivos.

Las cualidades primarias de estos materiales se aúnan a técnicas constructivas tradicionales y reiteradas, debidas a la falta de conocimiento de técnicas modernas, por parte de los constructores, o bien de su desinterés por aplicarlas, puesto que los métodos que han usado les han resultado eficaces dentro de la comunidad. " (Sánchez Lara. 1980:12)

No obstante, el nuevo orden político y religioso sí trajo consigo la conquista, una reordenación urbana alrededor de las nuevas construcciones religiosas y de gobernación, de estilo europeo, que tenía como propósito facilitar el control español del nuevo territorio. Al provocar de este modo la migración a la ciudad se provocó una alta desaparición de construcciones vernáculas y con ello su entendimiento de los procesos constructivos y de la región.

El siguiente gran periodo de dificultad para la arquitectura vernácula mexicana, a la que López Morales ya define como "La que nació de un lento y decantado proceso histórico en el cual mezcla elementos indigenas, africanos $y$ europeos" (López Morales. 1987:12), es cuando el entorno deja de ser la razón principal de existir de la arquitectura. Alejándose de los procesos y necesidades primarias, J. Taylor; "Los arquitectos anónimos de la historia, ante la escasez de recursos, tuvieron que desarrollar por necesidad formas arquitectónicas naturales muy prácticas y económicas que se enraizaban más en principios lógicos que en modas o caprichos pasajeros" (Taylor. 1984:9), potenciando este proceso mediante dos factores que se desarrollaron de forma exponencial en este periodo. La mercantilización de un derecho universal como es la vivienda, donde procesos de capitalización y especialmente la especulación ha convertido la construcción y diseño en una de las industrias más potentes a nivel mundial. Mientras que en consonancia con esta mercantilización debemos volver la 
vista al trabajo de arquitectos, diseñadores e ingenieros, que especialmente durante esta segunda mitad del siglo XX abrazaron de forma masiva como panacea la globalización de la arquitectura, con origen en la primera mitad del mismo siglo en parte de Europa y Estados unidos uniformizando lenguajes, materiales e imagen a nivel mundial.

Llegando al momento actual, habiendo recogido esta la evolución del siglo $\mathrm{XX}$ en aspectos de soluciones globales y lenguaje internacional, en un marco en el que los problemas de América latina se caracterizan por “(...) el progresivo envejecimiento de la población, el insuficiente dinamismo en la generación de empleo, la relativa reducción de la pobreza pero el aumento de la desigualdad en varios paises (...)" (CEPAL y PNUMA. 2002:47).

De este modo, aterrizando en el mundo de la edificación, tendencias como la Geek ha maximizado la inclusión de aditivos tecnológicos en un modelo arquitectónico fuertemente marcado por el abuso hacia la tecnología, como único medio para incrementar la sostenibilidad de las edificaciones.

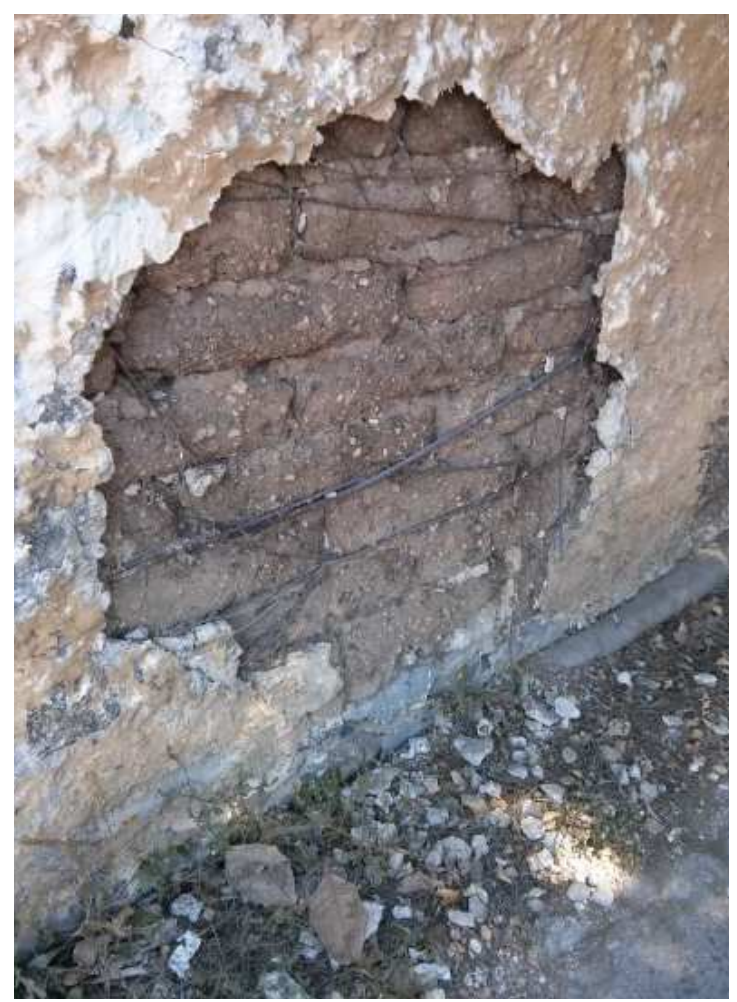

Fig. 2. Detalle de muro. Fuente. Fotografía propiedad del señor Alfred Esteller.
Se consolida de este modo el consumismo de la sociedad contemporánea en la arquitectura, donde “(...) la importancia del lugar, del espacio, de la materialidad y de la vivencia pasan a segundo plano, y en que lo importante es consumir arquitectura del mismo modo que gaseosas o seriales de TV." (Klotz. 2006:55), como analiza M. Klotz, al que es necesario añadir la sostenibilidad, pero ya no en forma de concepto, cómo define acertadamente T.H. Russ "una forma de llevar a cabo una actividad económica sensata desde el punto de vista medioambiental para obtener los resultados socialmente deseados" (Russ. 2010:61).

Para efectos del proceso de diseño, se transforma gran parte de la metodología de diseño actual donde se sobre-explotan los recursos naturales y se devuelven emisiones y residuos.

\section{Problemática}

El progreso tecnológico, del que "Hemos heredado (...), además de sus múltiples ventajas, un sentido antitético de lo práctico, sabedores de que disponemos de medios artificiales para suplir la ineficiencia..." (Taylor. 1984:9). La realidad es que a pocos, especialmente profesionales del gobierno de ciudades, de la planeación urbana y la construcción, realmente les interesa realizar acciones verdaderamente social, medioambiental y económicamente viables, que son, los pilares de la sostenibilidad. A este efecto, organizaciones no gubernamentales recogen la responsabilidad, pero así mismo las privadas, y son estas quienes han acogido el mercado para hacer un sistema capitalizado de certificaciones, la actualmente más conocida, LEED. "México aparece en la lista por primera vez y ocupa el noveno lugar. Al cierre del 2017, México contó con más de 305 proyectos certificados LEED" (Stanley, 2018). Y no solo LEED, como se menciona en la investigación desarrollado en la UNAM (Vallejo, sf).Y aunque se cuenten con programas nacionales como Programa de Certificación de Edificaciones Sustentables (PCES), NMX-AA164-SCF1-2013 de Edificación Sustentable, Sí Se Vive, del Infonavit y estos se encuentren en constante renovación, resulta insuficiente por los alcances de cada uno de los instrumentos. 


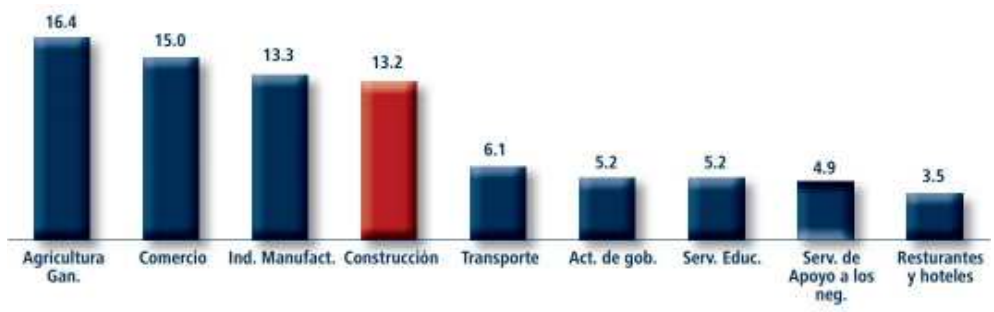

Fig. 3. Porcentajes activos de las industrias en México. Fuente. Cámara Mexicana de la Industria de la Construcción. CMIC.

\section{Justificación}

Ante esta gran necesidad, que surgen una serie de indicadores de sostenibilidad para México, casos como el desarrollado por estudiantes del Tec de Monterrey (Rodríguez, Campoy, Cantú y Orihuela, 2015) aplicables desde el sector de la industria de la construcción y urbanización hasta la planeación de las ciudades y la renovación urbana, que van desde el proceso de obtención de recursos materiales (materia prima) hasta que se termina la vida útil de los inmuebles. Es importante comprender que los indicadores de sostenibilidad son una herramienta para cuantificar la eficiencia con la que se desarrolla el proceso constructivo y vida útil del mismo una vez finalizado y que para ello, es de suma importancia conocer y poder cuantificar una serie de elementos que son necesarios en cada apartado de dichos indicadores para poder obtener un resultado lo más apegado a la realidad. Esta lista de indicadores sirve también a manera de guía durante el proceso constructivo, ya que, si no se desea evaluar, puede servir para de manera paramétrica tener claras ciertas líneas a considerar durante el proceso edificatorio, es esta relación la que se muestra en el siguiente apartado. Este sistema puede ser sin problema alguno integrado a las metodologías actuales de planeación y aportarnos muchos beneficios en ahorro energético y optimización de recursos.

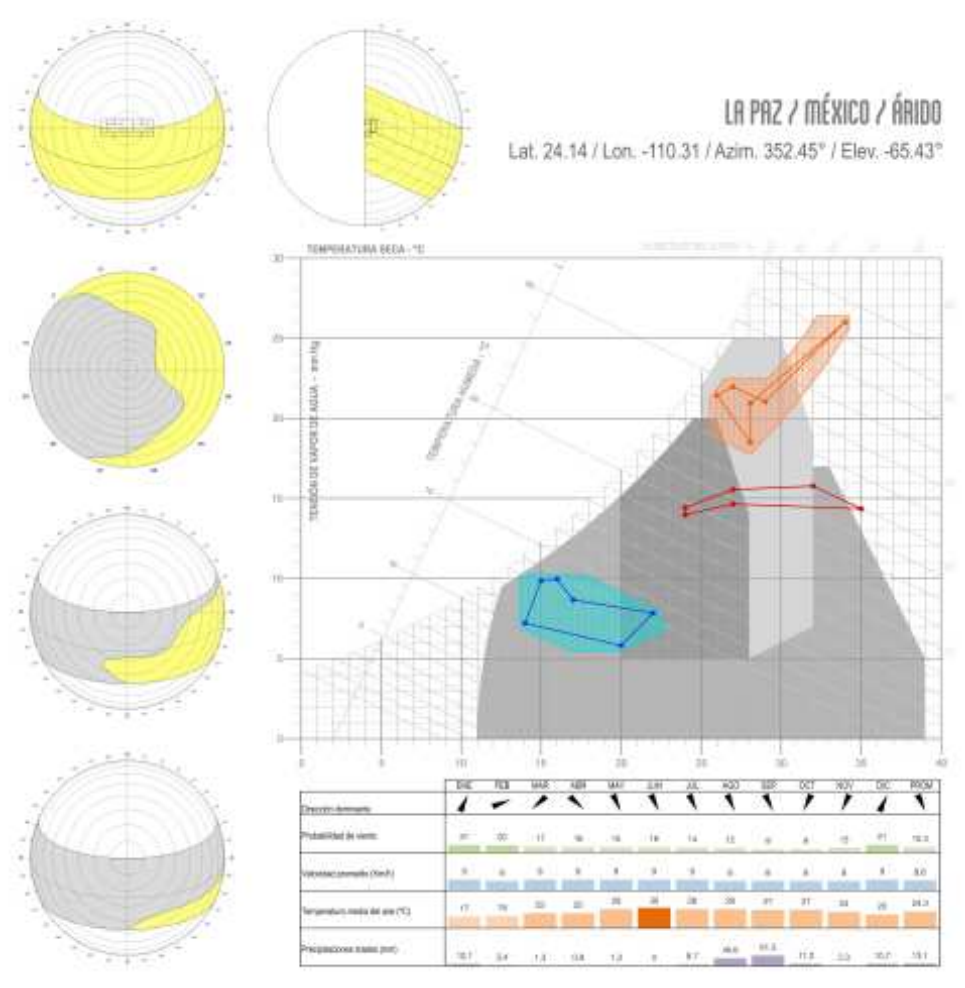

Fig. 4. Ficha de análisis climático Ficha climática y solar para un proyecto en la ciudad de La Paz, BCS, México. Fuente. Elaboración propia. 


\section{Producto}

1. Utilización de recursos materiales.

\subsection{Obtención de materias primas}

1.1.1 Cantidad de materias primas extraídas en la obtención del material.

1.1.2 Disponibilidad global de las materias primas necesarias.

1.1.3 Disponibilidad local de las materias primas necesarias.

1.1.4 Origen de los residuos y emisiones generadas para la obtención del material.

1.1.5 Cantidad de residuos y emisiones generadas en la obtención de las materias primas para la obtención del material.

1.1.6 Características físicas de los residuos y emisiones generadas en la obtención de las materias primas para la obtención del material.

1.1.7 Características químicas de los residuos y emisiones generadas en la obtención de las materias primas para la obtención del material.

1.1.8 Aprovechamiento de los residuos y emisiones generadas en el proceso de obtención de las materias primas para la obtención del material.

1.1.9 Tratamiento de los residuos y emisiones generadas en el proceso de obtención de las materias primas para la obtención del material.

1.1.10 Cantidad de materia prima reutilizada en el proceso de transformación del material.

1.1.11 Cantidad de material reutilizado externo al proceso de transformación del material.

1.1.12 Cantidad de material reutilizado dentro del proceso de transformación.

1.1.13 Cantidad de material reciclado externo al proceso de transformación del material.

1.1.14 Cantidad de material reciclado dentro del proceso de transformación.

\subsection{Proceso de producción del material}

1.2.1 Origen de los residuos y emisiones generadas en el proceso de producción del material.

1.2.2 Cantidad de residuos y emisiones generadas en el proceso de producción del material.

1.2.3 Características físicas de los residuos $\mathrm{y}$ emisiones generadas en el proceso de producción del material.

1.2.4 Características químicas de los residuos y emisiones generadas en el proceso de producción del material.

1.2.5 Aprovechamiento de los residuos y emisiones generadas en el proceso de obtención del material.

1.2.6 Tratamiento de los residuos y emisiones generadas en el proceso de obtención del material.

1.2.7 Características de la energía incorporada en la obtención del material

1.2.8 Consumo energético en la obtención del material.

1.2.9 Porcentaje de energía renovable utilizada en la obtención del material.

1.2.10 Adecuación del modelo energético con el proceso de obtención del material.

1.2.11 Impacto socioeconómico del proceso energético utilizado en el contexto global.

1.2.12 Impacto socioeconómico del proceso energético utilizado en el contexto local.

1.2.13 Impacto ambiental del proceso energético utilizado en el contexto global.

1.2.14 Impacto ambiental del proceso energético utilizado en el contexto local

1.2.15 Consumo energético en la obtención del material.

1.2.16 Porcentaje de energía renovable utilizada en la obtención del material.

1.2.17 Adecuación del modelo energético con el proceso de obtención del material.

1.2.18 Impacto socioeconómico del proceso energético utilizado en el contexto global.

1.2.19 Impacto socioeconómico del proceso energético utilizado en el contexto local.

1.2.20 Impacto ambiental del proceso energético utilizado en el contexto global.

1.2.21 Impacto ambiental del proceso energético utilizado en el contexto local

1.3 Repercusiones económicas y sociales de la producción del material

1.3.1 Costo económico de la producción del material.

1.3.2 Repercusiones sociales e impacto durante la obtención de materia prima. 


\subsection{Características del material finalizado}

1.4.1 Cantidad de material utilizado en la construcción del elemento construido.

1.4.2 Durabilidad del material.

1.4.3 Cantidad de emisiones nocivas para el medio ambiente generadas durante la vida útil del material.

1.4.4 Cantidad de emisiones nocivas para el ser humano generadas durante la vida útil del material.

1.4.5 Capacidad de reutilización del material con el mismo uso.

1.4.6 Capacidad de reutilización del material con diferente uso.

1.4.7 Capacidad de reparación del material.

1.4.8 Capacidad de adaptación del material.

1.4.9 Capacidad de reciclabilidad del material

1.5 Utilización del material (sistemas de construcción)

1.5.1 Cantidad de energía necesaria para el proceso de construcción de cada sistema.

1.5.2 Origen de los residuos generados en el proceso de construcción de cada sistema.

1.5.3 Cantidad de residuos generados en el proceso de construcción de cada sistema.

1.5.4 Características físicas de los residuos $\mathrm{y}$ emisiones generados en el proceso de construcción de cada sistema.

1.5.5 Características químicas de los residuos y emisiones generados en el proceso de construcción de cada sistema.

1.5.6 Aprovechamiento de los residuos $\mathrm{y}$ emisiones generadas en el proceso de construcción de cada sistema.

1.5.7 Tratamiento de los residuos y emisiones generadas en el proceso de construcción de cada sistema.

1.5.8 Durabilidad del sistema de construcción.

1.5.9 Coste económico de mantenimiento del sistema de construcción.

1.5.10 Capacidad del sistema de construcción para recuperar elementos individuales.

1.5.11 Capacidad del sistema de construcción para reparar elementos individuales.

1.5.12 Capacidad del sistema de construcción para ampliarse.
1.5.13 Capacidad del sistema de construcción para reconfigurar sus elementos.

1.5.14 Origen de los residuos y emisiones generados en el proceso de mantenimiento de cada sistema.

1.5.15 Cantidad de residuos y emisiones generadas en el proceso de mantenimiento de cada sistema.

1.5.16 Características físicas de los residuos $\mathrm{y}$ emisiones generados en el proceso de mantenimiento de cada sistema.

1.5.17 Características químicas de los residuos y emisiones generados en el proceso de mantenimiento de cada sistema.

1.5.18 Aprovechamiento de los residuos $\mathrm{y}$ emisiones generadas en el proceso de mantenimiento de cada sistema.

1.5.19 Tratamiento de los residuos y emisiones generadas en el proceso de mantenimiento de cada sistema.

1.6 Gestión del material como residuo

1.6.1 Porcentaje de elementos aprovechables tras el derribo del elemento construido.

1.6.2 Tratamiento de los residuos y emisiones producidos durante el derribo del elemento construido.

1.6.3 Capacidad de reintegración en la naturaleza tras la vida útil del material.

1.6.4 Coste energético de la destrucción del material.

1.6.5 Coste energético del reciclaje del material.

1.6.6 Cantidad de residuos y emisiones generados en el proceso de destrucción del material.

1.6.7 Características físicas de los residuos $\mathrm{y}$ emisiones generadas en el proceso de destrucción del material.

1.6.8 Características químicas de los residuos y emisiones generadas en el proceso de destrucción del material.

\section{Desarrollo de proceso edificatorio}

2.1 Relación entre el diseño del elemento construido y su emplazamiento

2.1.1 Nivel de aprovechamiento del entorno físico en el diseño del elemento construido. 
2.1.2 Nivel de aprovechamiento de los componentes climáticos en el diseño del elemento construido.

2.1.3 Nivel de utilización de estrategias de diseño para el aprovechamiento de agua.

2.1.4 Nivel de utilización de tecnologías para el aprovechamiento de agua.

2.1.5 Nivel de utilización de estrategias de diseño para la producción de alimentos.

2.1.6 Nivel de utilización de tecnologías para la producción de alimentos.

2.1.7 Nivel de utilización de estrategias de diseño para el desarrollo de la flora local.

2.1.8 Nivel de utilización de tecnologías para el desarrollo de la flora local.

2.1.9 Nivel de utilización de estrategias de diseño para el desarrollo de la fauna local.

2.1.10 Nivel de utilización de tecnologías para el desarrollo de la fauna local.

2.1.11 Impacto ambiental de los materiales utilizados en la construcción del elemento construido para el contexto local.

2.1.12 Impacto ambiental de los materiales utilizados en la construcción del elemento construido para el contexto regional.

2.1.13 Impacto ambiental de los materiales utilizados en la construcción del elemento construido para el contexto global.

2.1.14 Impacto socioeconómico de los materiales utilizados en la construcción del elemento construido para el contexto local.

2.1.15 Impacto socioeconómico de los materiales utilizados en la construcción del elemento construido para el contexto regional.

2.1.16 Impacto socioeconómico de los materiales utilizados en la construcción del elemento construido para el contexto global.

2.1.17 Impacto ambiental de la construcción del elemento construido para el contexto local.

2.1.18 Impacto ambiental de la construcción del elemento construido para el contexto regional.

2.1.19 Impacto socioeconómico de la construcción del elemento construido en el contexto local.

2.1.20 Impacto socioeconómico de la construcción del elemento construido en el contexto regional.

2.1.21 Nivel de adecuación histórico-cultural del elemento construido con el contexto local.

2.1.22 Nivel de adecuación histórico- cultural del elemento construido con el contexto regional.

2.1.23 Impacto socioeconómico del elemento construido en el contexto local.

2.1.24 Impacto socioeconómico del elemento construido en el contexto regional

2.2 Diseño constructivo y ejecución del elemento construido

2.2.1 Nivel de adecuación funcional de los materiales a su uso en la edificación.

2.2.2 Nivel de adecuación entre la solución constructiva y su función.

2.2.3 Nivel de relación entre la solución constructiva adoptada en el edificio y las capacidades industriales del emplazamiento a nivel local.

2.2.4 Nivel de relación entre la solución constructiva adoptada en el edificio y las capacidades industriales del emplazamiento a nivel regional.

2.2.5 Nivel de adaptabilidad funcional del diseño del elemento construido.

2.2.6 Nivel de resiliencia del diseño del elemento construido.

2.2.7 Nivel de durabilidad del diseño del elemento construido.

2.2.8 Nivel de reparabilidad de los componentes del elemento construido.

2.2.9 Capacidad de restauración y rehabilitación del elemento construido

\subsection{Consumo energético}

2.3.1 Cantidad de energía utilizada en el transporte del material.

2.3.2 Cantidad de energía utilizada en el transporte de mano de obra.

2.3.3 Cantidad de energía utilizada en el transporte de maquinaria.

2.3.4 Consumo energético en la ejecución del elemento construido por la mano de obra.

2.3.5 Consumo energético en la ejecución del elemento construido por la maquinaria.

2.3.6 Consumo energético en la ejecución del elemento construido por el transporte interno.

2.3.7 Porcentaje de energía renovable en la ejecución total del elemento construido 


\subsection{Coste económico}

2.4.1 Cantidad de recurso económico utilizado en el transporte del material.

2.4.2 Cantidad de recurso económico utilizado en el transporte de mano de obra.

2.4.3 Cantidad de recurso económico utilizada en el transporte de maquinaria.

2.4.4 Coste económico por la mano de obra.

2.4.5 Coste económico por el consumo energético de ejecución del elemento construido

\subsection{Residuos y emisiones}

2.5.1 Cantidad de residuos y emisiones generadas durante el diseño y ejecución del elemento construido (excluidos los procesos propios de los materiales y sistemas de construcción).

2.5.2 Aprovechamiento de los residuos y emisiones generadas en el diseño y ejecución del elemento construido (excluidos los procesos propios de los materiales y sistemas de construcción).

2.5.3 Tratamiento de los residuos y emisiones generadas en el diseño y ejecución del elemento construido (excluidos los procesos propios de los materiales y sistemas de construcción)

\section{Vida útil}

\subsection{Consumo energético}

3.1.1 Cantidad de energía consumida en la accesibilidad al edificio.

3.1.2 Consumo energético del elemento construido en uso.

3.1.3 Porcentaje de energía renovable del elemento construido en uso.

3.1.4 Cantidad de energía consumida por el edificio cuando no está en funcionamiento

\subsection{Equipamiento tecnológico}

3.2.1 Utilización de equipamiento tecnológico en iluminación.

3.2.2 Consumo energético en utilización de equipamiento tecnológico en iluminación.

3.2.3 Coste económico en utilización de equipamiento tecnológico en iluminación.

5.3.2.4 Nivel de mantenimiento en equipamiento tecnológico de iluminación.

3.2.5 Durabilidad del equipamiento tecnológico de iluminación.

3.2.6 Nivel de afección a la salud en equipamiento tecnológico de iluminación.

3.2.7 Utilización de equipamiento tecnológico en climatización.

3.2.8 Consumo energético en utilización de equipamiento tecnológico en climatización.

3.2.9 Coste económico en utilización de equipamiento tecnológico en climatización.

3.2.10 Nivel de mantenimiento en equipamiento tecnológico de climatización.

3.2.11 Durabilidad del equipamiento tecnológico de climatización.

3.2.12 Nivel de afección a la salud en equipamiento tecnológico de climatización.

3.2.13 Utilización de equipamiento tecnológico en aprovechamiento de agua.

3.2.14 Consumo energético en utilización de equipamiento tecnológico en aprovechamiento de agua.

3.2.15 Coste económico en utilización de equipamiento tecnológico en aprovechamiento de agua.

3.2.16 Nivel de mantenimiento en equipamiento tecnológico aprovechamiento de agua.

3.2.17 Durabilidad del equipamiento tecnológico aprovechamiento de agua.

3.2.18 Nivel de afección a la salud en equipamiento tecnológico aprovechamiento de agua.

3.2.19 Utilización de equipamiento tecnológico en producción de alimentos.

3.2.20 Consumo energético en utilización de equipamiento tecnológico en producción de alimentos.

3.2.21 Coste económico en utilización de equipamiento tecnológico en producción de alimentos.

3.2.22 Nivel de mantenimiento en equipamiento tecnológico producción de alimentos.

3.2.23 Durabilidad del equipamiento tecnológico producción de alimentos.

5.3.2.24 Nivel de afección a la salud en equipamiento tecnológico producción de alimentos.

3.2.25 Utilización de equipamiento tecnológico en seguridad. 
3.2.26 Consumo energético en utilización de equipamiento tecnológico en seguridad.

3.2.27 Coste económico en utilización de equipamiento tecnológico en seguridad.

3.2.28 Nivel de mantenimiento en equipamiento tecnológico de seguridad.

3.2.29 Durabilidad del equipamiento tecnológico de seguridad.

3.2.30 Nivel de afección a la salud en equipamiento tecnológico de seguridad.

3.2.31 Utilización de equipamiento tecnológico en automatismos.

3.2.32 Consumo energético en utilización de equipamiento tecnológico en automatismos.

3.2.33 Coste económico en utilización de equipamiento tecnológico en automatismos.

3.2.34 Nivel de mantenimiento en equipamiento tecnológico de automatismos.

3.2.35 Durabilidad del equipamiento tecnológico de automatismos.

3.2.36 Nivel de afección a la salud en equipamiento tecnológico de automatismos.

3.2.37 Utilización de equipamiento tecnológico en generación de energía.

3.2.38 Consumo energético en utilización de equipamiento tecnológico en generación de energía.

3.2.39 Coste económico en utilización de equipamiento tecnológico en generación de energía.

3.2.40 Nivel de mantenimiento en equipamiento tecnológico de generación de energía.

3.2.41 Durabilidad del equipamiento tecnológico de generación de energía.

3.2.42 Nivel de afección a la salud en equipamiento tecnológico de generación de energía.

3.2.43 Utilización de equipamiento tecnológico en sistemas de control y medición.

3.2.44 Consumo energético en utilización de equipamiento tecnológico en sistemas de control y medición.

3.2.45 Coste económico en utilización de equipamiento tecnológico en sistemas de control y medición.

3.2.46 Nivel de mantenimiento en equipamiento tecnológico de sistemas de control y medición.

3.2.47 Durabilidad del equipamiento tecnológico de sistemas de control y medición.
3.2.48 Nivel de afección a la salud en equipamiento tecnológico de sistemas de control y medición

\subsection{Residuos y emisiones}

3.3.1 Cantidad de residuos y emisiones producidos durante la vida útil del elemento construido.

3.3.2 Origen de los residuos y emisiones producidos durante la vida útil del elemento construido.

3.3.3 Porcentaje de residuos y emisiones tratados durante la vida útil del elemento construido.

3.3.4 Nivel de tratamiento de los residuos y emisiones producidos durante la vida útil del elemento construido.

3.3.5 Cantidad de residuos y emisiones producidos durante el derribo del elemento construido (excluidos los propios de materiales y sistemas ya contemplados)

\subsection{Bienestar humano}

3.4.1 Nivel de satisfacción funcional del usuario.

3.4.2 Nivel de satisfacción sensitiva del usuario.

3.4.3 Satisfacción sensorial para el usuario.

3.4.4 Nivel de calidad del aire interior.

3.4.5 Nivel de adecuación de las condiciones higrotérmicas del aire interior.

3.4.6 Nivel de adecuación de la velocidad de aire en el interior del elemento construido.

3.4.7 Cantidad de partículas en suspensión.

3.4.8 Nivel de ruido en el interior del elemento construido.

3.4.9 Nivel de radiación dentro del elemento construido.

3.4.10 Nivel de peligrosidad de la radiación dentro del elemento construido.

3.4.11 Nivel de adecuación de la cantidad de iluminación natural en el edificio.

3.4.12 Nivel de adecuación de la cantidad de iluminación artificial en el edificio

\section{Discusión}

Muchas empresas hoy en día se dedican a la consultoría ambiental, la mayoría de ellas, 
basan la calificación de las edificaciones mediante sistemas de certificación extranjeros y por los cuales se tiene que asumir un costo desproporcionalmente alto, asegurando así una asesoría durante todo el proceso de construcción por el equipo del cual se haya contratado la certificación, ya que el contratante, en ningún momento puede tener acceso a la información con la cual se está realizando la metodología de calificación, como resultado se entrega una letra que representa una calificación en el propio baremo creado por la empresa.

Poder contar con herramientas que no solo nos ayuden a facilitar el trabajo, sino que nos ayuden a canalizar las metas en cuanto a sostenibilidad supone un gran avance tanto para el diseñador, planeador, gobernante, pequeño o gran constructor. Además, este tipo de herramientas aportan de manera indirecta un compromiso y una vinculación interdisciplinaria.

El incremento de la demanda por parte de los usuarios por este tipo de metodologías generará en un mediano y largo plazo que el tema de evaluación sostenible en la edificación pueda extenderse y sobre todo especializarse hacia los parámetros y condicionantes de la arquitectura y construcción en México y así mismo fortalecer el vínculo entre cultura y política con las herramientas técnicas para marchar de manera integral hacia una meta en conjunto.

\section{Conclusiones}

La mirada hacia la sostenibilidad hoy en día está integrada por tres pilares cuyo objetivo es generar un crecimiento equitativo y justo. Sin embargo, para que las ciudades puedan desarrollarse conveniente en el mundo actual, es necesario echar mano de otros aspectos que enriquecen y fortalecen la labor. La gestión como parte de nuestro rol profesional es ahora necesario para la conformación de equipos de trabajo competente y preparado para las exigencias actuales. Es por ello la exigencia actual de incorporar nuevas herramientas, sistemas, procesos y metodologías que nos ayuden y complementen este proceso fortalecido de avance en la industria de la construcción.
La evolución adecuada de la ciudad, debe ser responsabilidad de un grupo interdisciplinario con la capacidad de adaptarse y resolver situaciones que hoy en día son fundamentales para la mejora del sistema de diseño y planeación. Es importante generar conciencia al respecto, pues para lograr una mayor eficiencia en los procesos es imprescindible considerarlo desde el principio de todo proyecto; sin importar la escala, los alcances en materia de sostenibilidad deben ser fijados como parte de los objetivos. En el caso de los indicadores, bien lo describen esta parte del proceso, tomando en cuenta las condicionantes del emplazamiento desde el primer momento. La consolidación de una metodología de Indicadores de Sostenibilidad depende del compromiso no solo de los profesionistas hacia un sistema que nos permite tomar decisiones de manera equilibrada con bases cuantificables, sino que representa un compromiso por parte de instituciones, gobierno y particulares, generando así una red de trabajo con una meta en común.

Debemos entonces nosotros como profesionales convertirnos en agentes activos del cambio, fomentando y generando conocimiento $\mathrm{y}$ herramientas locales que permitan la generación de procesos de diseño y edificatorios apegados a la realidad planetaria.

\section{Referencias}

Sánchez Lara, R. M. (1980) "El significado de la Arquitectura Vernácula." En: Secretaría de Educación Pública Instituto Nacional de Bellas Artes. (Ed.). Arquitectura Vernácula. Cuadernos de arquitectura y conservación del patrimonio artístico. (pp 11-17). (Primera edición) México, Litorarte México.

Taylor, J. (1984) Arquitectura anónima. Una visión cultural de los principios prácticos del diseño. (Primera edición). España. Editorial Stylos.

López Morales, F. J. (1987) Arquitectura Vernácula en México. (Primera edición) México. Editorial Trillas.

Cámara Mexicana de la Industria de la Construcción, CMIC. (2011) Los Retos de la Infraestructura en México 2013-2018. Disponible en CMIC website: http://www. 
cmic.org/cmic/economiaestadistica/2013/

Retos.pdf

Comisión Económica para América Latina y el caribe (CEPAL) y Programa de las Naciones Unidas para el Medio Ambiente. (2002) La sostenibilidad del desarrollo en América Latina y el Caribe: desafíos y oportunidades. (Primera edición). Chile. Editorial de las Naciones Unidas.

Klotz, M. (2006) "Arquitectura para el consumo". ARQ (Santiago), n. 62, 54-56. Chile. Editorial Pontificia Universidad Católica de Chile.

Russ, T.H. (2010) Sustainability and Design ethics. (Primera edición). Gran Bretaña. Editorial CRC Press Taylor \& Francis Group.

Stanley, S. (2018) México hace su debut en la lista anual de los 10 primeros países y regiones para LEED. USA. Green Building Certification Institute Online. Disponible en https://gbci.org/m\%C3\%A9xico-hacesu-debut-en-la-lista-anual-de-los-10principales-pa\%C3\%ADses-y-regionespara-leed

Vallejo, V., s.f. Las diversas certificaciones aplicables a los edificios sustentables en México. Multidisciplin@, Revista electrónica de la Facultad de Estudios Superiores Acatlán, No. 18. México. Universidad Autónoma de México Online http://revistas.unam.mx/index.php/ multidisciplina/article/view/50693/45451

Rodriguez, S., Campoy, M., Cantu, E., Orihuela, E. (2015) Propuesta de modelo integral de evaluación sostenible de la vivienda social en México. Ambiente costruido, vol (15), No. 4. México. https://doi.org/10.1590/ s1678-86212015000400036

Aion Plan; Arquitectura Sostenible (2014), Indicadores de sostenibilidad en la construcción. Disponible en Aion Plan website: www.aionplan.com 